\title{
In vivo platelèt aggregation and plasma catecholamines in acute myocardial infarction
}

\begin{abstract}
In vivo platelet aggregation assessed with the Filtragometer and potential correlates were compared among (1) patients with acute myocardial infarction (AMI), (2) normal controls, (3) patients with acute chest pain in whom AMI was eventually ruled out (ROMI), and (4) chronic outpatients (Cardiac Clinic group) with a history of myocardial infarction and/or angina pectoris. The measure was independent of sex, age, platelet count, immediate food intake, serum cholesterol, and triglyceride levels. The AMI group showed higher in vivo platelet aggregation than any of the other three groups $(p<0.01)$. Least in vivo aggregation was seen in the normal group. Despite lack of correlation with the platelet aggregation measure, plasma epinephrine and norepinephrine showed statistically significant differences between the AMI and each of the other three groups. Our data support an association between platelet function and AMI, although not necessarily a cause and effect relationship. (Am HEART J 104:1255, 1982.)
\end{abstract}

Richard P. Sorkin, M.D., Joyce M. Tokarsky, B.S., Marla J. Huber-Smith, B.S., Jeanne F. Steiger, and Daisy S. McCann, Ph.D. Westland and Ann Arbor, Mich.

The precise contribution of platelet function to the development of myocardial infarction remains to be defined. Many in vitro attempts to assess subtle differences in platelet activity from one individual to another are plagued with potential artifacts. Among these difficulties are the probable loss of the most fragile platelets incurred in the process of preparing platelet rich plasma, the use of exogenous aggregating agents, and the removal of the platelets from the influence of their intravascular environment. ${ }^{1}$

Hornstra and ten Hoor's Filtragometer ${ }^{2}$ obviates some of the difficulties encountered by the conventional measurements of platelet activity and has been developed into a commercially available, compact instrument. We therefore set out to determine the relationship between this measure of what has been labelled as an "in vivo" platelet aggregation and acute myocardial infarction as well as with a number of plasma factors which might influence it. Statistically significant differences in this platelet

From the Department of Medicine, Wayne County General Hospital; and the Cardiology Division, Medicine; and Biological Chemistry of the University of Michigan, School of Medicine.

Supported by grants-in-aid from the Michigan Heart Association and the Medical Staff Research and Education Fund, Wayne County General Hospital.

Received for publication March 30, 1981; revision received June 8, 1981; accepted June 29, 1981.

Reprint requests: Daisy S. McCann, Ph.D., Wayne County General Hospital, Depl. of Medicine, P.O. Box 549, Wayne, MI 48184. function were observed between patients with acute myocardial infarction and (1) normal controls, (2) patients with acute chest pain in whom acute myocardial infarction was subsequently ruled out, and (3) outpatient Cardiac Clinic patients with a history of myocardial infarction and/or angina pectoris. The relationships between in vivo platelet aggregation and serum cholesterol, triglyceride, and plasma catecholamine levels were tested.

\section{METHODS}

Platelet aggregation measurement. The measurement of in vivo platelet aggregation was made with the commercially available Filtragometer (Fleischman Electronics Corp., Montclair, N.J.) following the manufacturer's instructions. The instrument measures the concentration of preformed platelet aggregates in the subject's circulation by recording the time required for those aggregates to occlude a $20 \mu \mathrm{m}$ nickel filter as defined by development of a $5 \mathrm{~mm} \mathrm{Hg}$ pressure differential across the filter. ${ }^{2}$ The system was first rinsed with $3.8 \%$ citrate. A 19 gauge butterfly needle was then inserted into the subject's antecubital vein with every effort made to minimize vein wall trauma. Twenty-five IU heparin per milliliter of blood were infused into the tubing immediately adjacent to the butterfly needle. The rate of withdrawal of blood was maintained constant at $2 \mathrm{ml} / \mathrm{min}$ by use of a Harvard pump. Twenty milliliters of blood was withdrawn for platelet counts and chemical analysis. The tubing was then connected to the mixing chamber of the instrument from which the heparinized whole blood was drawn through the nickel filter. If occlusion did not occur within 600 seconds, the run was terminated and the result 
Table I. Interassay reproducibility of catecholamine pools each measured the indicated number of times over a 2 - to 3-month period

\begin{tabular}{lllcc} 
Pool & Catechol & $N$ & Mean \pm 1 S.D. & $\begin{array}{c}\text { \% coefficient } \\
\text { variation }\end{array}$ \\
\hline \multirow{2}{*}{40181} & NE & 13 & $444.7 \pm 36.5$ & 8.2 \\
& E & 12 & $398.0 \pm 51.7$ & 12.9 \\
73080 & NE & 14 & $361.0 \pm 30.5$ & 8.4 \\
& E & 15 & $30.6 \pm 14.9$ & 48.6 \\
\multirow{4}{4}{1381} & NE & 10 & $339.2 \pm 39.4$ & 11.6 \\
& E & 10 & $55.1 \pm 18.0$ & 32.7 \\
& NE & 12 & $216.0 \pm 29.4$ & 13.6 \\
& E & 10 & $32.6 \pm 11.4$ & 35.0 \\
\hline
\end{tabular}

$\mathrm{NE}=$ norepinephrine; $\mathrm{E}=$ epinephrine.

recorded as "over 600 seconds." (Such values are rare in nonmedicated persons and were not incorporated into the statistical analysis.)

Long-term reproducibility of platelet aggregation was assessed by repeated measurements, two to eight per subject, performed over a 2- to 32 -week period in 10 normal controls. Short-term reproducibility and the effect of food intake on aggregation was evaluated in eight control subjects who were studied in the fasting state between 9:00 and 10:00 A.M., 2 and 6 hours after a breakfast consisting of 2 eggs, bacon, toast and coffee; and 24 hours later, again in the fasting state. To confirm that occlusion of the nickel filter is a function of platelet aggregates rather than of fibrin clots, the occluding material was examined microscopically using Wright's stain. The response of the Filtragometer measure to the ingestion of $260 \mathrm{mg}$ of aspirin was assessed in 12 normal subjects.

Catecholamine determination. Chemical data included serum cholesterol, triglyceride, and plasma catecholamine levels. The latter were determined by our modification ${ }^{3-5}$ of the Passon and Peuler single isotope derivative technique. ${ }^{6}$ The intra-assay reproducibility for this procedure is $7.1 \%$ and $12.7 \%$ for norepinephrine and epinephrine, respectively. The interassay reproducibility is in part a function of the catecholamine concentrations (Table I) and should be reported for normal concentrations, not high concentrations $>600 \mathrm{pg} / \mathrm{ml}$, as they often are. Another consideration in reporting catecholamine levels is the orthostatic position of the subject just prior to and during the period the blood is drawn. Norepinephrine means \pm standard deviation for normal controls in our laboratory are $235 \pm 51,333 \pm 78$, and $424 \pm 100 \mathrm{pg} / \mathrm{ml}$ for supine $(\mathrm{n}=34)$, seated $(\mathrm{n}=30)$, and standing $(\mathrm{n}=11)$ subjects. The analogous plasma epinephrine concentrations are $33 \pm 18,48 \pm 34$, and $54 \pm 30 \mathrm{pg} / \mathrm{ml}$. Blood for plasma catecholamines was placed into cooled heparinized vacutainers containing glutathione $(5 \mathrm{mM}$ final concentration in blood). The blood and chemicals were mixed gently by inversion and centrifuged in the cold as soon as possible. While plasma catecholamines are stable stored frozen, they do not tolerate repeated freeze-thaw cycles, and no specimen assayed in this study was submitted to more than one such cycle.

Study groups. The following study groups were defined. $I$-group $N=27$ normal subjects without history or objective evidence of cardiovascular disease were drawn from our laboratory and hospital staff. II-group $\mathrm{C}=23$ Cardiac Clinic patients ( 3 female, 20 male) who reported for medical follow-up at 3-month intervals because of a recent history of myocardial infarction and/or angina pectoris (age $55 \pm 11$ years, mean $\pm \mathrm{SD}$ ). III-group $A M I=14$ patients ( 4 female, 10 male) on admission to the Cardiac Care Unit in whom acute myocardial infarction (AMI) was subsequently confirmed by (1) a typical clinical history of acute chest pain, (2) evolving ECG changes diagnostic of acute myocardial infarction, and (3) elevated total plasma creatine kinase (CK) with positive $\mathrm{MB}$ bands (age $59 \pm 11$ years). Five of 14 patients had diabetes mellitus and 5 of 14 had a prior history of hypertension. $I V$-group $R O M I=16$ patients (3 female, 13 male) on admission to the Cardiac Care Unit with acute chest pain in whom acute myocardial infarction was subsequently ruled out, based on the negative data obtained from the criteria outlined for the AMI group above (age $56 \pm 10$ years). Four of 16 patients were diabetic, 5 of 16 had a prior history of hypertension. $V$-group $1 w k A M I=\operatorname{six}$ patients from group III above (two female, four male) in whom repeat studies were obtained 1 week after the acute myocardial infarction episode. The small number of patients in this group reflects the fact that the majority of patients at this point were on medications which interfere with platelet aggregation studies, plasma catecholamine analysis, or both (age $65 \pm 6$ years).

Patients for groups AMI and ROMI were admitted to the study on a random basis within the following selection process. Any patient admitted into the Cardiac Care Unit with acute chest pain between the hours of 8:00 A.M. and 4:00 P.M. On weekdays was included in the study provided: (1) he/she gave informed consent to participation in the study and (2) he/she could assure us that no known antiplatelet drug (aspirin, indomethacin, ibuprofen, dipyridamole, and sulfinpyrazone) had been taken during the preceding 5 days. The need for informed consent skewed the AMI population to a preponderance of less complicated infarction, i.e., Killip class I and II. Admission to the Cardiac Clinic group (group C) was limited because the majority of this population was on drugs known or thought to affect platelet aggregation.

From the point of view of catecholamine analysis it is important to note that in groups $\mathrm{N}$ and $\mathrm{C}$ blood was drawn from seated individuals, whereas in groups AMI, ROMI, and 1 wk AMI blood was obtained from patients in the supine position. We therefore included in the data normal plasma norepinephrine and epinephrine values for 15 subjects aged $31 \pm 6$ years in the basal state, i.e., 7 A.M. fasting and still supine after 7 hours bed rest (group B).

Statistics. The data were submitted to statistical analysis of variance using the MIDAS program of the Univer- 


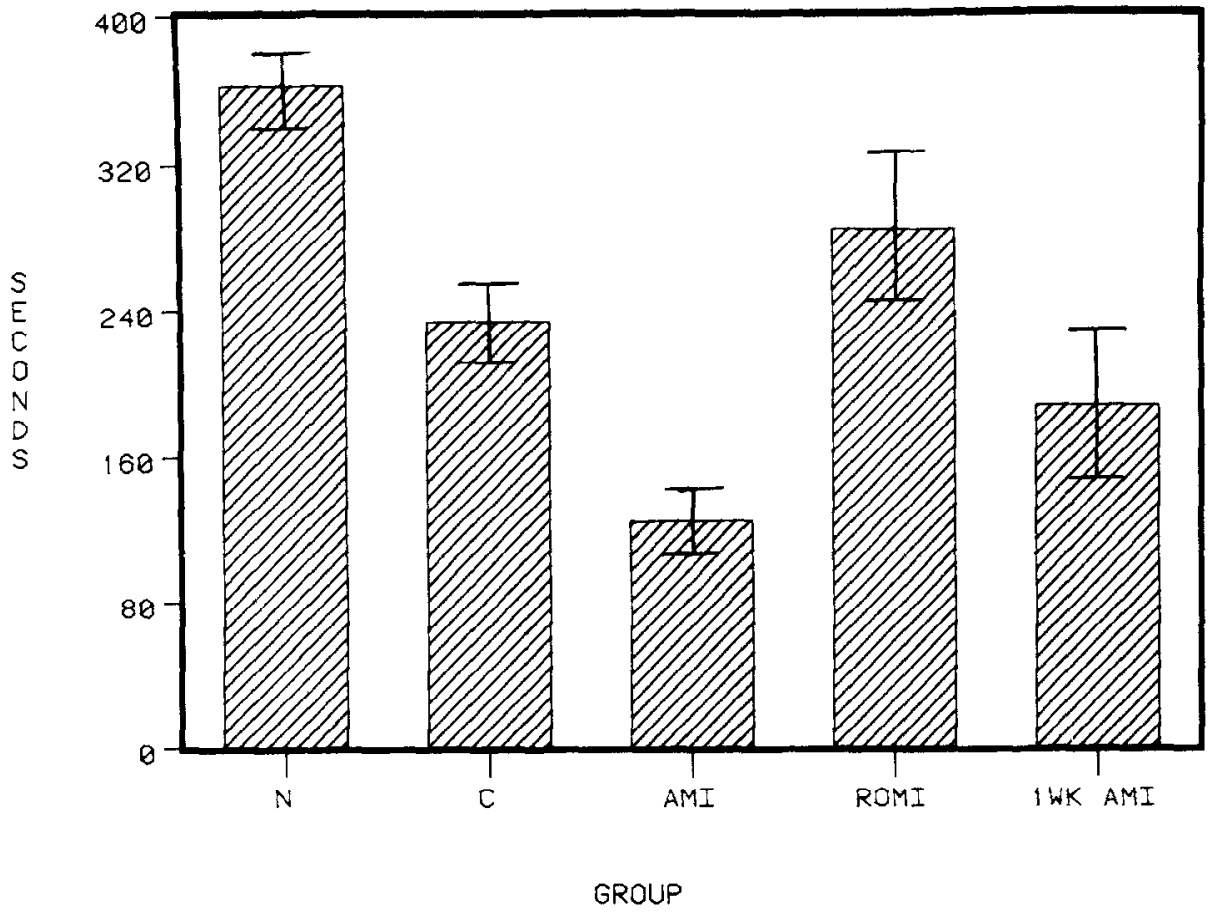

Fig. 1. In vivo platelet aggregation (mean \pm S.E.M.) of the Normal $(N)$, Cardiac Clinic (C), Acute Myocardial Infarction ( $A M I)$, Myocardial Infarction Ruled Out (ROMI) and 1 week post-Myocardial Infarction (1wk $A M I)$ groups.

sity of Michigan's MTS computer. The group I wk AMI was eliminated from the computer analysis because the number of cases studied was small. The data are included in this report because they are indicative of the course followed by in vivo platelet aggregation subsequent to the acute event.

\section{RESULTS}

Control aggregation data. In vivo platelet aggregation data for 27 normal controls, 14 men and 13 women, averaged $363 \pm 23$ seconds (mean \pm SEM unless noted otherwise) to filter occlusion time. There was no significant difference between the males ( $n=14$, age $39 \pm 13$ [mean \pm S.D.] years, platelet aggregation $375 \pm 30$ seconds) and the females ( $n=13$, age $42 \pm 11$ years, platelet aggregation in seconds to filter occlusion time $350 \pm 35$ ).

For 10 normal controls, the reproducibility of the measure assessed by repeated measurements (two to eight per subject performed over a 2- to 32-week period) was $21.8 \%$ (coefficient of variation). Reproducibility of the measure for fasting subjects between 9:00 and 10:00 A.M. on two consecutive mornings was $19.5 \%$. Reproducibility within a given 24-hour period was not affected by food intake or time of day and was $18.5 \%$ for seven subjects. Data from the normal subjects were tested for bias related to sex and/or age. In these 27 cases no significant differences could be attributed to either of those two variables. Microscopic inspection of the occluding material using Wright's stain confirmed that the mass consisted of platelets with some entrapped red and white blood cells. Twenty-four hours after ingestion of $260 \mathrm{mg}$ of aspirin, 12 normal subjects showed an average increase of $42.2 \%$ in their filter occlusion time and hence a proportional decrease in their in vivo platelet aggregation.

Platelet counts. Among the individuals tested, only one had a frankly abnormal platelet count of 739,000 platelets $/ \mathrm{mm}^{3}$. The filter occluded at 71 seconds; the case was not included in the statistical analysis. Platelet counts for the Normal, AMI, ROMI, and Cardiac Clinic groups were 288,000 $\pm 16,600$; $288,000 \pm 27,700 ; 246,000 \pm 19,200 ;$ and $260,000 \pm$ 14,200 per $\mathrm{mm}^{3}$, respectively. Over the platelet count range 112,000 to $483,000 / \mathrm{mm}^{3}$, no statistically significant relationship was apparent between count and in vivo aggregation.

Platelet aggregation data among groups. Comparisons of in vivo platelet aggregation between the groups studied are shown in Fig. 1. Individual changes in in vivo platelet aggregation between the time of the AMI and 1 week later are shown in Fig. 2. Significant differences at $p<0.01$ confidence levels 


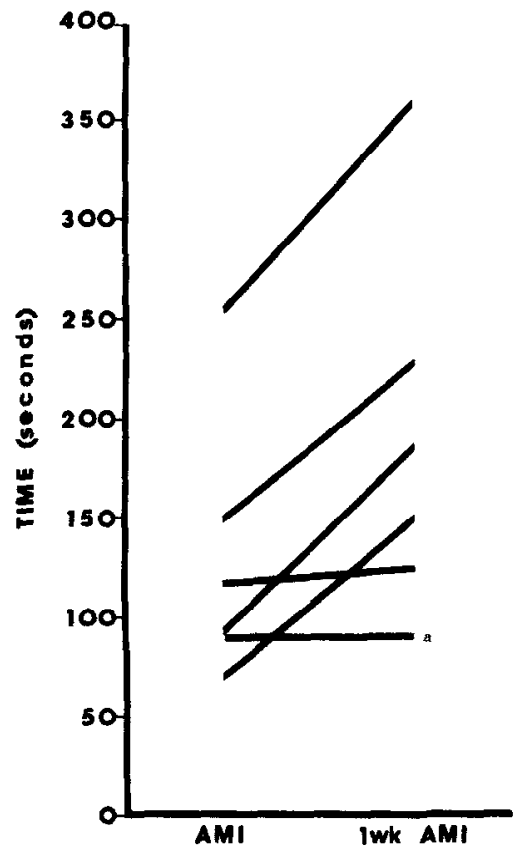

Fig. 2. Individual changes in in vivo platelet aggregation between the acute phase of myocardial infarction and 1 week later. Patient $(a)$ reinfarcted and died 1 week after the second Filtragometer measurement.

existed between the AMI group and each of the other groups. Substantially lower aggregation (longer time required for occlusion) than for any other group was displayed by the Normal control group $(p<0.05)$.

Blood lipids. Mean cholesterol levels for the Normal, AMI, ROMI, and Cardiac Clinic groups were $234 \pm 13,225 \pm 19,229 \pm 27$, and $244 \pm 11 \mathrm{mg} / \mathrm{dl}$, respectively. Triglyceride levels in these groups were $149 \pm 19,242 \pm 35,199 \pm 38$, and $208 \pm 18 \mathrm{mg} / \mathrm{dl}$, respectively. No significant correlations were seen between in vivo platelet aggregation and serum cholesterol or triglyceride levels.

Plasma catecholamines. The plasma catecholamine analysis showed significantly higher norepinephrine as well as epinephrine concentrations for the AMI group as compared to the other groups (Fig. 3). Despite the high catecholamine levels of the AMI group, there was no statistically significant correlation between either catecholamine levels individually or their sum and platelet aggregation.

\section{DISCUSSION}

The Filtragometer appears to measure a real platelet function since (1) its nickel filter is occluded by platelets at the termination of the measure, (2) it responds as anticipated to aspirin inhibition of platelet activity, (3) it is relatively independent of immediate physiological fluctuations of plasma con- stituents such as lipid concentrations and (4) it is reproducible albeit at a coefficient or variation of $\pm 19 \%$.

Over a decade ago, Jörgensen et al. ${ }^{7}$ demonstrated that transient platelet aggregates in the microcirculation of the myocardium of animals can cause arrhythmias and sudden death. Discovery of the roles played by thromboxane ( $\mathrm{TXA}_{2}$ ) and prostacyclin $\left(\mathrm{PGI}_{2}\right)$ in platelet aggregation and vascular tissue physiology have increased the expectation that platelets play a crucial role in atherogenesis and its sequelae.$^{8,9}$ However, direct evidence of this in the human disease has been difficult to formulate. ${ }^{10}$

Previous studies. In part this may be due to the "in vitro" techniques generally used to assess platelet functions, which of necessity neglect to consider certain platelet populations as well as the interaction of platelets with vessel wall constituents and/or other formed elements of blood. Wu and Hoak ${ }^{11}$ have attempted to circumvent the problem by formalin fixing the formed elements of blood and then comparing the platelet count of a fixed aliquot of blood with that of an unfixed one. Using this technique they reported substantial increases in circulating platelet aggregates in acute myocardial infarction, acute cerebral vascular insufficiency, and acute peripheral arterial insufficiency as compared to normal controls, patient controls, patients with stable angina, and chronic peripheral arterial insufficiency. Reports by other authors with this technique have been contradictory, some supporting $\mathrm{Wu}$ and Hoak's findings, ${ }^{12,13}$ some unable to do so, ${ }^{14,15}$ and some ambivalent. ${ }^{16}$ In a later publication $\mathrm{Wu}$ and Hoak ${ }^{17}$ confirmed their earlier findings by studying similar populations with a "spontaneous" platelet aggregation technique.

Steele et al. ${ }^{18}$ using conventional platelet aggregation response curves over the range 0.5 to $2.0 \mu \mathrm{g}$ $\mathrm{ADP} / \mathrm{ml}$, did not see significant differences between their controls and 21 men with chronic coronary artery disease. A number of other workers have used platelet aggregation response thresholds to serially diluted aggregating agents, usually ADP, to demonstrate changes in platelet activity from normal control subjects to patients with cerebral vascular disease, ${ }^{19,20}$ ischemic heart disease, ${ }^{21}$ angina pectoris, ${ }^{22}$ and acute myocardial infarction..$^{23} \mathrm{~A}$ cautionary note at the end of Zahavi and Dreyfuss' discussion ${ }^{23}$ suggests that other acute stress situations such as stroke, pulmonary infarction, and acute severe infection may produce similar aggregation patterns to those observed in acute myocardial infarction.

Increased platelet aggregation in AMI. Among the 


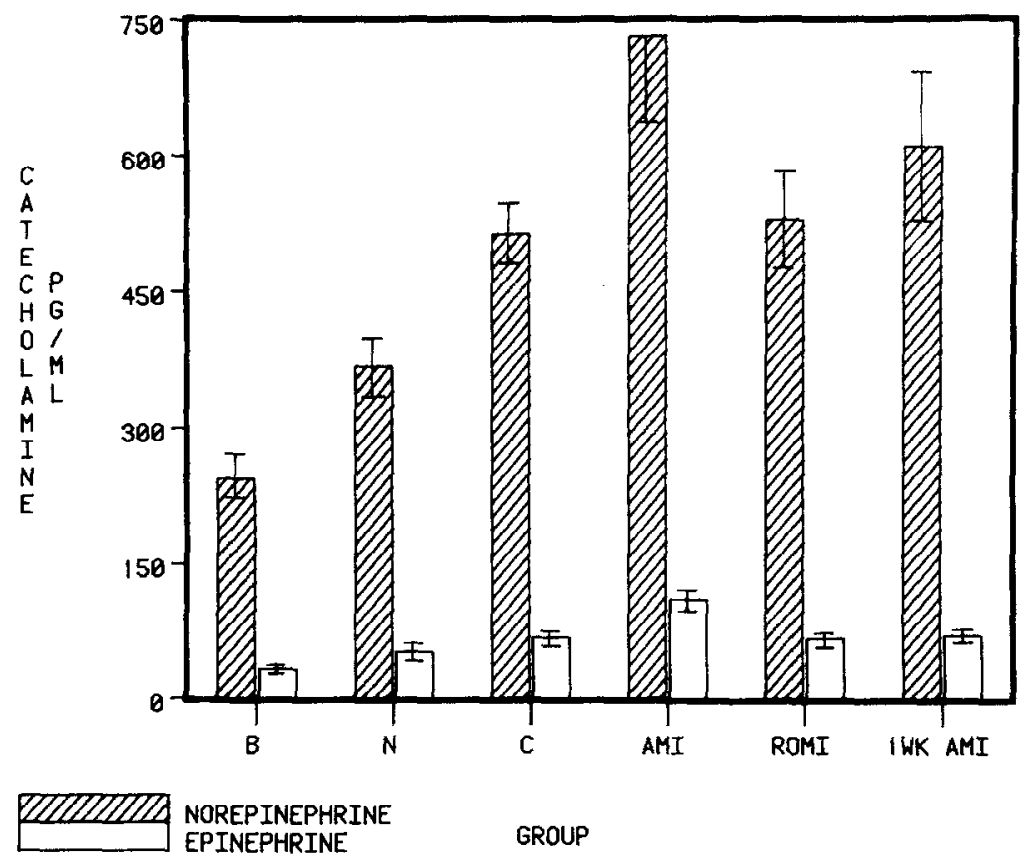

Fig. 3. Mean plasma epinephrine and norepinephrine concentrations of the groups studied. $B$ represents the group drawn in the basal state (see text); for the other abbreviations see legend for Fig. 1. Blood was drawn from supine individuals for the basal, AMI, ROMI, and 1 wk AMI groups and in the seated position for the Normal and Cardiac Clinic groups.

relatively few data which have been published using Hornstra and ten Hoor's Filtragometer are several lipid studies ${ }^{24-26}$ and a report of increased in vivo platelet aggregation in 15 patients with cardiovascular events, 10 with AMI and five with acute cerebrovascular accidents. ${ }^{27}$ Our Filtragometer data support the concept that increased in vivo platelet aggregation is associated with AMI and is reflected not only at the site of the occlusion but in the peripheral circulation as well. Despite the significant difference obtained when the AMI group is compared with the other groups, there is some overlap in individual values. This is especially true between the AMI and the ROMI groups. Thus, even though the ROMI group is a high risk group (in our population 14 out of 16 had a prior history of cardiovascular disease and/or systemic hypertension), it is nonetheless doubtful that high in vivo platelet aggregation is the cause of myocardial infarction, but does suggest that it may be a contributory factor to the course of a multifactorial disease.

Plasma composition effects. Shortly before the interactions between platelet aggregation and $\mathrm{TXA}_{2}$ and $\mathrm{PGI}_{2}$ were elucidated, both Fleischman et al. ${ }^{24,25}$ and Hornstra et al. ${ }^{26}$ reported that replacement of approximately 500 calories per day of saturated with unsaturated fats $(60 \%$ linoleate, $\mathrm{C} 18: 2)$ resulted in lowered in vivo platelet aggregation. We did not look into the potential of dietary manipulation of in vivo platelet aggregation but were interested in the immediate effect of feeding vs fasting, since we wanted to study AMI and ROMI groups as soon after admission to the Cardiac Care Unit as possible without consideration of recent food intake. We saw no difference. These data agree with the conclusion reached by Carvalho et al..$^{28}$ that in vivo platelet aggregation is a function of the platelets rather than of the immediate plasma composition. Specifically these authors demonstrated that platelets of patients with type II hyperlipoproteinemia aggregated to one twenty-fifth of the mean concentration of epinephrine and one third of the threshold dose of ADP and collagen, as compared to normal controls. Patients with type IV hyperlipoproteinemia did not show platelet hypersensitivity despite elevated serum triglyceride levels. Carvalho et al. ${ }^{28}$ were not able to induce abnormal aggregation behavior in normal platelets by 30 to 60 minute incubation with type II plasma. Platelet fatty acid composition may, however, play a role in hyperaggregability and myocardial infarction. ${ }^{29-31}$ Shattill et al. ${ }^{30}$ and Stuart et al. ${ }^{31}$ have demonstrated hyperaggregability of platelets after incubation with lipid liposomes in which the cholesterol/phospholipid ratio was adjusted to 
2.2:1, a condition which in vitro raises the platelet cholesterol content.

Johnson et al. ${ }^{32,33}$ have reported sex-linked differences of platelet aggregation in animals and man. Among their human population these same authors showed increasing platelet responsiveness with increasing donor age. Measurement of preformed platelet aggregates in our normal group of 14 men and 13 women showed neither a sex- nor an agerelated bias.

Consideration of catecholamines. Comparisons between plasma catecholamine levels were made between our Normal and Cardiac Clinic groups, since both were drawn seated; and between our Basal, AMI, and ROMI groups, since these were all drawn supine. The latter two groups were of particular interest since they received identical treatment in terms of admission to and subsequent care in the hospital. No difference was seen between the Normal and the Clinic groups but significant differences exist between the AMI and the ROMI groups $(p<0.025)$ for both norepinephrine and epinephrine, and between either of these two groups and the Basal group $(p<0.001)$. The difference in catecholamine levels between the AMI and the ROMI groups must be ascribed to a difference in physiological stress between the two groups; emotional stress is presumably similar since the diagnosis of AMI was not established or ruled out at the time our studies were performed. Despite the similarities between aggregation and catecholamine data in terms of differences between the various groups, no statistically significant correlation was obtained between the two measures. Correlation between the Filtragometer results and the catecholamine data were not altered by the removal of six patients who had received drugs designed to interact with catecholamine receptors. Videback et al. $^{34}$ were among the first to report elevation of total plasma catecholamines to $1000 \pm 450 \mathrm{pg} / \mathrm{ml}$ (mean \pm S.D.) in AMI. This figure compares reasonably well with ours, where the mean sum of plasma norepinephrine plus epinephrine was $845 \mathrm{pg} / \mathrm{ml}$. The same is true of the mean control values, where we obtained $235 \mathrm{pg} / \mathrm{ml}$ compared to Videback's $243 \mathrm{pg} / \mathrm{ml}$. Videback et al..$^{34}$ concluded that catecholamine levels were substantially below those which can induce arrhythmias in experimental myocardial infarction. They are also two to three orders of magnitude below threshold doses needed to promote platelet aggregation in in vitro systems.

Conclusions. The Filtragometer technique for assaying platelet aggregation is an interesting one because results are obtained immediately and with a minimum of sample manipulation. AMI probably has a multifactorial etiology. Coronary occlusion due to platelet aggregates may play a critical role for a substantial number of myocardial infarctions, vasospasm for others, and still unidentified physiological factors for the remainder. Our data support the concept of an association between platelet function and AMI. They do not support the concept of a necessary cause-and-effect relationship but are compatible with the hypothesis that in vivo platelet aggregation may contribute to the subsequent development and eventual size of the myocardial infarction. The role of early intervention with drugs which inhibit platelet aggregation remains an area for future investigation.

We thank Dr. Francisco Eng for stimulating our interest in this area of research.

\section{REFERENCES}

1. Hampton JR, Michell JRA: Human blood coagulation, haemostasis and thrombosis. 2nd ed. Oxford, 1976, Blackwell Scientific Publications, p 536.

2. Hornstra G, ten Hoor F: The filtragometer: A new device for measuring platelet aggregation in venous blood of man. Thromb Diath Haemorrh 34:531, 1975.

3. Michaels R, Huber-Smith MJ, McCann DS: Evaluation of transcendental meditation as a method of reducing stress. Science 192:1242, 1976.

4. Lederman RP, McCann DS, Work B: Endogenous plasma epinephrine and norepinephrine in last trimester of pregnancy and labor. Am J Obstet Gynecol 129:5, 1977.

5. Eng FWHT, Huber-Smith MJ, McCann DS: The role of sympathetic activity in normal renin essential hypertension. Hypertension 2:14, 1980.

6. Passon PG, Peuler JD: A simplified radiometric assay for plasma norepinephrine and epinephrine. Anal Biochem 51:618, 1973.

7. Jörgensen L, Rowsell HC, Hovig T, Glynn MF, Mustard JF: Adenosine diphosphate induced platelet aggregation and myocardial infarction in swine. Lab Invest 17:616, 1967.

8. Dusting GJ, Moncada S, Vane JR: Prostaglandins, their intermediates and precursors: Cardiovascular actions and regulatory roles in normal and abnormal circulatory systems. Prog Cardiovasc Dis 21:405, 1979.

9. Harker LA, Ritchie JL: The role of platelets in acute vascular events. Circulation 62(suppl V):13, 1980.

10. Schafer AI, Handin RI: The role of platelets in thrombotic and vascular disease. Prog Cardiovasc Dis 22:31, 1979.

11. Wu KK, Hoak JC: A new method for the quantitative detection of platelet aggregates in patients with arterial insufficiency. Lancet 2:924, 1974.

12. Mehta P, Mehta J: Platelet function studies in coronary artery disease. Evidence for enhanced platelet microthrombus formation activity in acute myocardial infarction. Am J Cardiol 43:757, 1979

13. Dougherty J, McIntyre N, Weksler BB: Assessment of platelet activation in relation to acute thrombotic events. Blood 46:1021, 1975 .

14. Prazich JA, Rapaport SI, Samples JR, Engler R: Platelet aggregate ratio-standardization of technique and test results in patients with myocardial ischemia and patients with cerebrovascular disease. Thromb Haemost 38:597, 1977.

15. Rohrer TF, Pfister B, Weber C, Imhof PR, Stucki P: Quantitative changes in platelet aggregation due to physiological and pathological factors and medication. Blut 36:21, 1978. 
16. Guyton JR, Willerson JT: Peripheral venous platelet aggregates in patients with unstable angina pectoris and acute myocardial infarction. Angiology 28:695, 1977.

17. Wu KK, Hoak JC: Spontaneous platelet aggregation in arterial insufficiency: Mechanisms and implication. Thromb Haemost 35:702, 1976.

18. Steele PP, Weily HS, Davies H, Genton E: Platelet function studies in coronary artery disease. Circulation 48:1194, 1973.

19. Dougherty JH, Levy DE, Weksler BB: Platelet activation in acute cerebral ischaemia. Lancet 1:821, 1977.

20. Walsh PN, Pareti FI, Corbett JJ: Platelet coagulant activities and serum lipids in transient cerebral ischemia. N Engl $J$ Med 295:854, 1976

21. Salky N, Dugdale M: Platelet abnormalities in ischemic heart discase. Am J Cardiol 32:612, 1973.

22. Frishman WH, Weksler B, Christodoulou JP, Glynn, MF, Mustard JF: Reversal of abnormal platelet aggregability and change in exercise tolerance in patients with angina pectoris following oral propranolol. Circulation 50:887, 1974.

23. Zahavi J, Dreyfuss F: An abnormal pattern of adenosine diphosphate induced platelet aggregation in acute myocardial infarction. Thromb Diath Haemorrh 21:76, 1969.

24. Fleischman AI, Justice D, Bierenbaum ML, Stier A, Sullivan A: Beneficial effect of increased dietary linoleate upon in vivo platelet function in man. J Nutr 105:1286, 1975.

25. Fleischman AI, Bierenbaum ML, Justice D, Stier A, Sullivan
A, Fleischman M: Titrating dietary linoleate to in vivo platelet function in man. Am J Clin Nutr 28:601, 1975.

26. Hornstra G, Lewis B, Chait A, Karvonen MJ, Lewis B, Turpeinen $\mathrm{O}$, Vergroesen AJ: Influence of dietary fat on platelet function in man. Lancet 1:1155, 1973.

27. Fleischman AI, Bierenbaum ML, Justice D, Stier A, Sullivan A: In vivo platelet function in acute myocardial infarction, acute cerebrovascular accidents and following surgery. Thromb Res 6:205, 1975.

28. Carvalho ACA, Colman RW, Lees RS: Platelet function in hyperlipoproteinemia. N Engl J Med 290:434, 1974.

29. Valles J, Aznar J, Santos MT: Platelet fatty acids in acute myocardial infarction. Thromb Res 14:231, 1979.

30. Shattil SJ, Anaya-Galindo R, Bennett J, Colman RW, Cooper RA: Platelet hypersensitivity induced by cholesterol incorporation. J Clin Invest 55:636, 1975.

31. Stuart MJ, Gerrard JM, White JG: Effect of cholesterol on production of thromboxane $\mathrm{B}_{2}$ by platelets in vitro. $\mathrm{N}$ Engl $J$ Med 302:6, 1980.

32. Johnson M, Ramey E, Ramwell PW: Androgen-mediated sensitivity in platelet aggregation. Am J Physiol:Heart Circ Physiol 1:H381, 1977.

33. Johnson M, Ramey E, Ramwell PW: Sex and age differences in human platelet aggregation. Nature 253:355, 1975.

34. Videback $J$, Christensen NJ, Sterndorff B: Serial determination of plasma catecholamines in myocardial infarction. Circulation 46:846, 1972.

NOTE:

After December 10th, Address Manuscripts to:

Dean T. Mason, M.D., Editor-in-Chief

AMERICAN HEART JOURNAL, Editorial Office

Cardiac Center, Cedars Medical Center

Cedars South $\rightarrow$ Suite 0

1295 N.W. 14th Street

Miami, Florida 33125 